\title{
COMPORTAMENTO SEXUAL E CARACTERÍSTICAS SEMINAIS DE SUÍNOS JOVENS COM DIFERENTES DESEMPENHOS DE CRESCIMENTO
}

\author{
SEXUAL BEHAVIOR AND SPERM CHARACTERISTICS IN YOUNG \\ BOARS WITH DIFFERENT GROWING PERFORMANCE
}

\author{
Fabiane Mendonça Ferreira ${ }^{1}$, Ivo Wentz ${ }^{2}$, Isabel Regina Scheid ${ }^{3}$, Simone Bonine Afonso $^{3}$, Antônio Lourenço \\ Guidoni $^{4}$ \& Fernando Pandolfo Bortolozzo ${ }^{2}$
}

\begin{abstract}
RESUMO
A capacidade reprodutiva dos machos é de grande importância na eficiência da indústria de criação animal. A investigação de características reprodutivas fornece informações sobre a idade ideal à primeira coleta de sêmen. $\mathrm{O}$ objetivo deste trabalho foi avaliar o comportamento sexual e as características seminais de machos suínos jovens com diferentes ganhos de peso do nascimento aos $100 \mathrm{~kg}$. Aos 143 dias de idade, 62 machos Landrace e Large White com 810 a 885g/dia (n=29) (grupo de desempenho 1; GD1) e com 649a 694g/dia (n=33)(GD2) foram avaliados quanto ao comportamento sexual. A partir de 150 dias, foi coletado sêmen de 33 destes machos duas vezes por semana durante onze semanas. As características seminais foram monitoradas até que os machos atingissem os 232 dias de idade. Machos GD2 tiveram melhor performance sexual que machos GD1 quando expostos a fêmeas em estro. Houve aumento do volume de sêmen, da concentração e da motilidade espermáticas e do número total de espermatozóides no ejaculado. Machos GD1 ejacularam maiores volumes de sêmen ( $\mathrm{p}=0,043$ ) que machos GD2, mas com maior percentual de espermatozóides anormais ( $\mathrm{p}=0,022)$. Machos GD2 ejacularam sêmen com maior concentração espermática ( $\mathrm{p}=0,027)$. Não foram encontradas diferenças entre os grupos de desempenho em relação à motilidade espermática $(\mathrm{p}=0,258)$, número total de espermatozóides ejaculados $(\mathrm{p}=0,195)$ e liberação diária de espermatozóides $(p=0,579)$. Estes dados indicam que machos com maior ganho de peso do nascimento aos $100 \mathrm{~kg}$ apresentam performance sexual inferior e, até atingirem 232 dias de idade, produção espermática similar a de machos com menores taxas de crescimento, no entanto com maior percentual de células espermáticas anormais.
\end{abstract}

Descritores: machos jovens, produção espermática, qualidade espermática.

\begin{abstract}
The reproductive capacity of males is of great importance in the efficiency of animal husbandry industry. The investigation of reproductive characteristics provides information about the ideal age at first semen collection. The aim of this work was to evaluate sexual behavior and seminal characteristics of young boars with different daily gain from birth to $100 \mathrm{~kg}$. With 143 days of age, 62 Landrace and Large White boars with 810 to $885 \mathrm{~g} /$ day $(\mathrm{n}=29)$ (performance group 1; PG1) and with 649 to $694 \mathrm{~g} /$ day (n=33) (PG2) were evaluated for sexual behavior. From 150 days, semen was collected from 33 of these boars twice per week during eleven weeks. The seminal characteristics were monitored until the boars were 232 days of age. The PG2 boars had a better sexual performance than PG1 boars when exposed to estrus females. There was an increasing in semen volume, sperm concentration and motility, and total sperm per ejaculate. The PG1 boars ejaculated higher semen volume $(p=0.043$ ) than PG2 boars, but with higher percent of abnormal spermatozoa $(\mathrm{p}=0.022)$. PG2 boars ejaculated semen with higher sperm concentration $(\mathrm{p}=0.027)$. No differences were found between performance groups in relation to sperm motility ( $\mathrm{p}=0.258)$, total number of spermatozoa per ejaculate $(\mathrm{p}=0.195)$, and daily sperm output $(\mathrm{p}=0.579)$. These data indicate that young boars with higher daily gain from birth to $100 \mathrm{~kg}$ show inferior sexual performance and, until reaching 232 days of age, similar sperm production, comparatively to boars with lower growing rate, however with higher percent of abnormal sperm cells.
\end{abstract}

Key words: young boars, sperm production, sperm quality.

${ }^{1}$ Fundação Universidade de Cruz Alta, Cruz Alta, RS. ${ }^{2}$ Setor de Suínos, Faculdade de Veterinária (FAVET) de Porto Alegre, Universidade Federal do Rio Grande do Sul (UFRGS). ${ }^{3}$ Médica Veterinária autônoma. ${ }^{4}$ Centro Nacional de Pesquisa de Aves e Suínos, EMBRAPA, Concórdia, S.C. CORRESPONDÊNCIA: F. M. Ferreira [e-mail: fabianemf@hotmail.com] Andrade Neves 308; 98000-000 Cruz Alta, RS - Brasil. 


\section{INTRODUÇÃO}

Uma parcela importante da eficiência reprodutiva do rebanho suíno depende do potencial de produção espermática de machos jovens. $\mathrm{O}$ atingimento da puberdade não significa necessariamente a aquisição da aptidão reprodutiva [2]. Via de regra, a recomendação para início da utilização de machos na reprodução é em torno dos oito meses de idade [8]. Isso por que, no período pós-púbere, os ejaculados caracterizam-se por uma quantidade relativamente pequena de células espermáticas e elevado percentual de alterações da morfologia espermática, limitando a utilização intensiva dessa categoria de animais. O objetivo deste trabalho foi investigar o comportamento sexual e as características seminais de machos suínos jovens com diferentes taxas de ganho de peso do nascimento até os $100 \mathrm{~kg}$.

\section{MATERIAIS E MÉTODOS}

\section{Animais e local}

O experimento foi realizado em uma unidade de melhoramento genético de suínos de uma agroindústria localizada no oeste catarinense. Foram selecionados ao acaso 62 machos das raças Landrace e Large White.

\section{Tratamentos}

Com base no ganho de peso diário (GPD) do nascimento até os $100 \mathrm{~kg}$ foram definidos dois grupos de desempenho: foram considerados do grupo de desempenho 1 (GD1) os animais que apresentaram taxas de ganho de peso diário (GPD) médias de 810 a 885 gramas por dia (Tratamento 1) e do grupo de desempenho 2 (GD2) os machos que tiveram GPD médio de 649 a 694 gramas (Tratamento 2). O ganho de peso diário (GPD) do nascimento até os $100 \mathrm{~kg}$ foi calculado através da divisão do peso $(\mathrm{kg})$ pela idade (d).

\section{Delineamento experimental}

Os animais foram distribuídos completamente ao acaso em baias individuais e procederam-se avaliações do comportamento sexual e de parâmetros seminais durante um período de onze semanas consecutivas.

\section{Experimento 1: Avaliação do Comportamento Sexual}

Após atingirem $100 \mathrm{~kg}$ de peso corporal e uma idade média de 143 dias, 29 machos do GD1 e 33 machos do GD2 (Tabela 1) foram submetidos a um teste para avaliação do comportamento sexual. Em uma baia de $3 \times 4 \mathrm{~m}$ cada macho foi colocado na presença de uma fêmea em estro por um período diário de 15 minutos. A cada apresentação, foi observado o número de saltos (montas) corretos e incorretos realizados pelo macho sobre a fêmea. O teste foi dado por concluído no momento em que o macho saltou corretamente sobre a fêmea, de maneira tal que possibilitasse a cópula (monta correta), não sendo permitida, no entanto, a realização da cobertura. Foi estabelecido um número máximo de dez exposições à fêmea com intervalos variáveis de 1 a 3 dias entre as apresentações.

\section{Experimento 2: Avaliação das Características Seminais}

\section{Coleta de Sêmen}

Após concluírem o teste de avaliação do comportamento sexual, 33 animais (GD1, $\mathrm{n}=17$; GD2, $\mathrm{n}=16$ ) foram treinados em manequim fixo para a coleta de sêmen pelo método da mão enluvada. Foi estabelecido um regime de duas coletas semanais de sêmen com intervalos regulares de 3 e 4 dias entre as coletas. Este regime de coleta de sêmen foi adotado até os animais atingirem a idade média de 232 dias.

\section{Análise do Ejaculado}

Terminada a coleta, era imediatamente avaliado o volume ejaculado. Em seguida, era feita uma estimação da motilidade espermática sob microscopia óptica de campo claro em 200 aumentos. Foram retiradas amostras de todos os ejaculados para a contagem do número de células espermáticas em Câmara Hemocitométrica (Neubauer) e determinação da concentração espermática.

A cada duas coletas de sêmen, foi realizado um exame da morfologia espermática sob microscopia de contraste de fase em 1000 aumentos. As alterações da morfologia espermática foram classificadas em defeitos de peça intermediária, colo, defeitos de cauda, cauda em laço, gota protoplasmática proximal, gota protoplasmática distal (GPD), defeitos teratológicos e de cabeça.

O número total de espermatozóides ejaculados foi calculado através da multiplicação da concentração espermática/mL de sêmen pelo volume total ejaculado por cada animal a cada coleta de sêmen. A estimação da liberação diária de espermatozóides (liberação diária de espermatozóides) foi realizada através do cálculo do número de SPTZ ejaculados a cada coleta de sêmen e a divisão do valor obtido pelo nú- 
mero de dias compreendido entre cada coleta de sêmen (três e quatro dias).

\section{Análise Estatística}

Para a comparação do número de montas corretas e incorretas utilizou-se o teste de qui-quadrado. Para a análise das variáveis foi utilizado o procedimento GLM, SAS [7]. Para o volume de sêmen, a motilidade, a concentração espermática, o número total de espermatozóides ejaculados e a morfologia espermática utilizou-se análise de variância para amostras repetidas considerando-se o efeito do grupo de desempenho e da semana de coleta. As médias foram comparadas pelo teste $t$ de Student.

\section{RESULTADOS}

Na Tabela 1 são apresentados os valores de ganho de peso diário médio até os $100 \mathrm{~kg}$, o peso dos animais e a idade média em que atingiram os $100 \mathrm{~kg}$.

\section{Experimento 1}

Todos animais saltaram sobre as fêmeas. Do total de 62 machos avaliados, somente dois não realizaram a monta correta. Os animais do GD1 e do GD2 apresentavam em média $133,43 \pm 1,01$ e $151,87 \pm 0,50$ dias de idade, respectivamente, havendo, portanto, uma diferença de 18,44 dias de idade entre os dois GD na primeira apresentação à fêmea. Os machos pertencentes ao GD2 apresentaram uma melhor performance quando expostos à presença da fêmea em cio comparativamente aos animais do GD1, caracterizada por um menor número de montas incorretas até se posicionarem corretamente sobre a fêmea (Tabela 2). Os machos do GD1 e do GD2 realizaram, respectivamente,

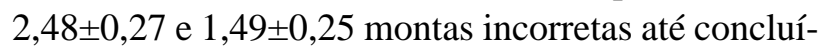
rem a avaliação do comportamento sexual.

\section{Experimento 2}

Os animais dos tratamentos GD1 e GD2 apresentavam em média 142,75 $\pm 15,67 ; 156,67 \pm 12,67$ dias de idade na primeira coleta de sêmen, respectivamente.

Considerando os valores médios obtidos em todo o período de coleta de sêmen, houve efeito do grupo de desempenho sobre o volume de sêmen produzido. O GD1 apresentou um volume médio de sêmen $(174,34 \pm 6,54 \mathrm{~mL})$ superior $(\mathrm{p}=0,0426)$ ao $\mathrm{GD} 2$ $(153,30 \pm 6,59 \mathrm{~mL})$ (Figura 1).

Não houve diferença significativa entre os machos do GD1 $(74,42 \pm 0,61 \%)$ e do GD2 $(75,48 \pm 0,62 \%)(\mathrm{p}=0,258)$ quanto à motilidade das células espermáticas.

Os animais do GD2 apresentaram concentração espermática média $\left(183,34 \pm 14,09 \times 10^{6} \mathrm{SPTZ} / \mathrm{mL}\right)$ superior ao GD1 $\left(135,64 \pm 14,03 \times 10^{6} \mathrm{SPTZ} / \mathrm{mL}\right)$ $(\mathrm{p}=0,0268)$.

Em relação à morfologia espermática (Figura 2), foi observado que os animais do GD1 apresentaram um porcentual médio superior de alterações espermáticas $(40,72 \pm 3,11 \%)$, comparativamente ao GD2 $(29,79 \pm$ $3,14 \%)(\mathrm{p}=0,0218)$. No decorrer do período de coleta de sêmen, foi observada uma redução gradual e paralela do porcentual de alterações espermáticas do sêmen de ambos os grupos de desempenho.

Não foram observadas diferenças significativas entre o GD1 $\left(24,32 \pm 2,02 \times 10^{9} \mathrm{SPTZ} /\right.$ ejaculado $)$ e o GD2 (28,28 $\left.\pm 2,03 \times 10^{9} \mathrm{SPTZ/ejaculado}\right)$ em relação ao número total de espermatozóides ejaculados

Tabela 1. Ganho de peso diário médio (GPD) até $100 \mathrm{~kg}$, peso corporal médio e idade média aos $100 \mathrm{~kg}$ dos machos dos grupo de desempenho 1 (GD1) e 2 (GD2) submetidos ao teste para avaliação do comportamento sexual.

\begin{tabular}{cccccccc}
\hline Animais & N & GPD (g/dia) & Erro padrão & Peso (kg) & Erro padrão & Idade (dias) & Erro padrão \\
\hline GD1 & 29 & 824,53 & 12,41 & 101,12 & 3,80 & 128,70 & 4,34 \\
GD2 & 33 & 668,08 & 13,26 & 98,62 & 2,16 & 147,62 & 1,31 \\
\hline
\end{tabular}

Tabela 2. Montas incorretas e corretas realizadas pelos machos dos grupos de desempenho (GD) 1 e 2 no teste de avaliação do comportamento sexual.

\begin{tabular}{|c|c|c|c|c|c|c|}
\hline \multirow{2}{*}{ GD } & \multicolumn{2}{|c|}{ Montas incorretas } & \multicolumn{2}{|c|}{ Montas corretas } & \multirow{2}{*}{$\begin{array}{c}\text { Total de } \\
\operatorname{montas}(n)\end{array}$} & \multirow{2}{*}{$\begin{array}{l}\text { Nível de probabilidade na } \\
\text { comparação entre os grupos }\end{array}$} \\
\hline & (n) & $(\%)$ & (n) & $(\%)$ & & \\
\hline 1 & 43 & 60,56 & 28 & 39,44 & 71 & \\
\hline 2 & 15 & 31,91 & 32 & 68,09 & 47 & 0,002 \\
\hline
\end{tabular}


$(\mathrm{p}=0,1950)$ (Figura 3) e à liberação diária de espermatozóides (GD1=17,24 $\pm 10,71$ X 109 $\mathrm{SPTZ} / \mathrm{dia}$; GD2=16,75 $\pm 10,11 \times 10^{9} \mathrm{SPTZ} /$ dia) durante todo o período de coleta de sêmen $(\mathrm{P}=0,5797)$.

\section{DISCUSSÃO}

\section{Experimento 1}

Neste trabalho, a performance dos machos pode ser considerada ótima, uma vez que, do total de 62 animais testados, somente dois não saltaram sobre as fêmeas em estro. Este desempenho foi bem superior ao observado por Nelssen et al. [6] que relataram que somente $65 \%$ dos machos de 210 dias saltaram sobre as fêmeas em estro. Os animais deste experimento foram criados em grupo até o momento de iniciarem o teste, então, é possível que a oportunidade de adquirir experiência sexual, através do contato físi-

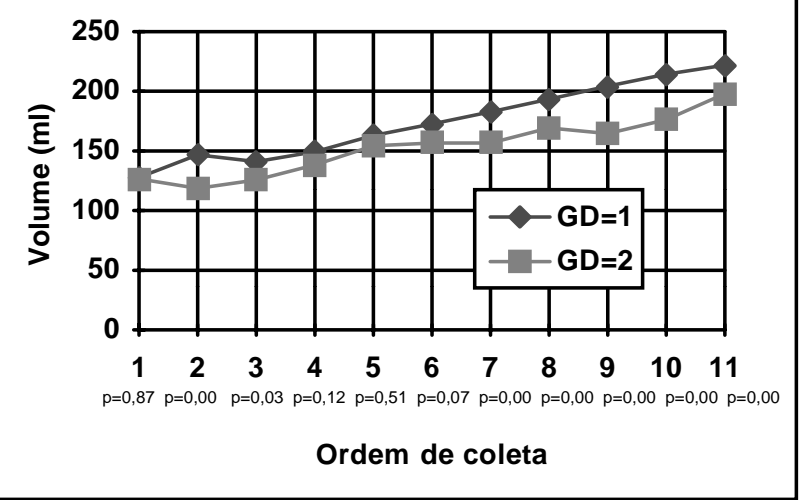

Figura 1. Variação no volume de sêmen de machos suínos dos grupos de desempenho (GD) 1 e 2 em função da ordem de coleta.

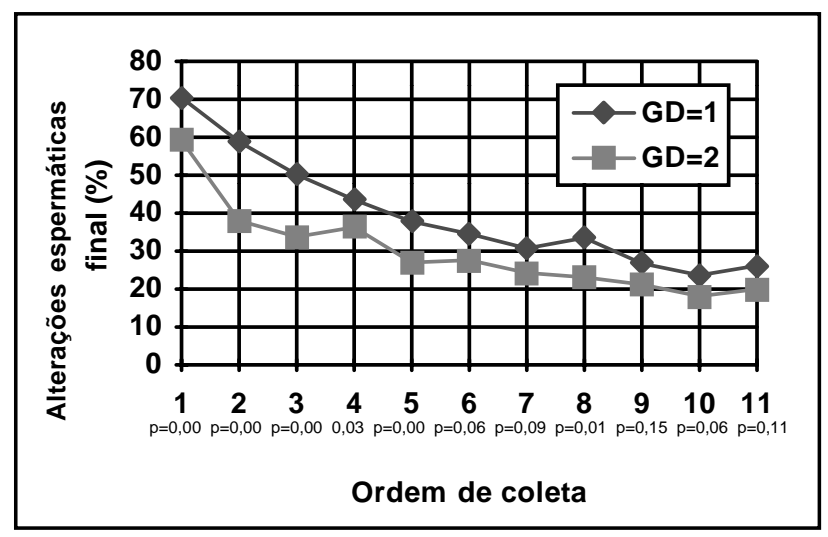

Figura 2. Variação da morfologia espermática de machos suínos dos grupos de desempenho (GD) 1 e 2 em função da ordem de coleta. co e das interações sociais durante a fase de crescimento, tenha contribuído para a expressão de um desempenho favorável durante a avaliação do comportamento sexual $[4,5]$. Como havia uma semelhança de tamanho corporal entre os animais dos dois grupos de desempenho, é possível assumir que a melhor performance dos machos do GD2 se deva à idade mais avançada destes em relação aos animais do GD2.

\section{Experimento 2}

No presente trabalho, o parâmetro fixado para o início do período experimental foi o atingimento dos $100 \mathrm{~kg}$ de peso corporal. Desta forma, foram avaliados animais que apresentavam diferentes idades ao longo das onze semanas durante as quais foram estudadas as características espermáticas.

$\mathrm{O}$ volume de sêmen tem importantes implicações na função reprodutiva do suíno. Na espécie suína, grandes volumes de sêmen são necessários para a estimulação mecânica das contrações uterinas. $\mathrm{O}$ volume de sêmen ejaculado pelos machos dos dois grupos de desempenho aumentou gradualmente durante o período experimental de coletas regulares de sêmen, não sendo possível observar, no entanto, uma tendência à estabilização desta variável. Um desenvolvimento mais acelerado das glândulas sexuais acessórias poderia explicar, em parte, o maior volume de sêmen produzido pelos animais do GD1, comparativamente ao GD2. É importante ressaltar que a quantidade de sêmen ejaculado pelos animais dos dois grupos de desempenho permaneceu acima de $100 \mathrm{~mL}$ desde a primeira coleta de sêmen, valor considerado o limite mínimo capaz de garantir bons resultados de fertilidade na espécie suína [9].

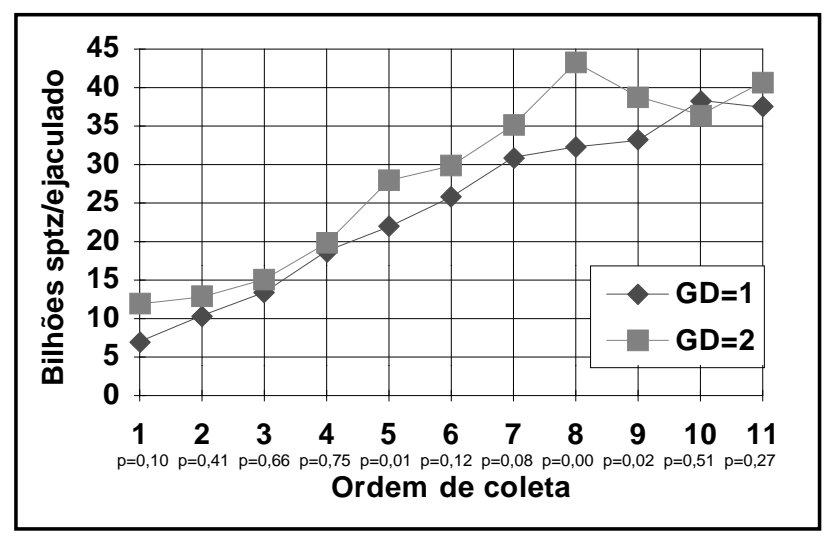

Figura 3. Número total de espermatozóides no ejaculado de machos suínos dos grupos de desempenho (GD) 1 e 2 em função da ordem de coleta. 
Foi possível verificar um aumento gradual da concentração espermática durante as primeiras sete semanas de coleta. Em torno dos 200 dias de idade pode ser observado o atingimento de valores relativamente constantes nos ejaculados dos dois grupos de desempenho. Como existe uma correlação positiva entre idade e concentração espermática [8], é possível que os valores superiores apresentados pelos machos do GD2 seja atribuída à idade mais avançada desses animais em relação ao GD1.

Os baixos valores do número total de espermatozóides ejaculados nas primeiras semanas de coleta de sêmen são característicos do período póspúbere [3]. A LDE proporciona uma estimativa de aproximadamente 83 a $88 \%$ da produção diária de espermatozóides. No presente estudo, a LDE aumentou acentuadamente entre os 150 e os 200 dias de idade, apresentando uma tendência à estabilização a partir dos 210 dias de idade.

Nas primeiras coletas foi observado um elevado percentual de alterações da morfologia espermática, o que não leva ao descarte, mas inviabiliza o uso dos reprodutores até a normalização desses valores. É importante ressaltar que machos do GD1 mantiveram, ao longo de todas as ordens de coleta, uma tendência de apresentar valores superiores de alteração espermática. A alta incidência de alterações morfológicas das células espermáticas observada nos animais dos dois grupos de desempenho reflete as condições imaturas da espermatogênese durante esta fase.

A análise geral dos parâmetros seminais revela um aumento progressivo do volume de sêmen, concentração espermática e do número total de espermatozóides ejaculados. À medida que avançou a idade dos machos, foi observada uma melhoria da qualidade espermática. Parece razoável supor que as diferenças encontradas nos parâmetros reprodutivos de animais com diferentes desempenhos de crescimento possam ser atribuídas à diferença de idade $\mathrm{e}$ provável estabelecimento mais tardio do processo espermatogênico nos animais mais jovens. É possível que essas diferenças iniciais venham a desaparecer num período posterior, uma vez que os machos podem apresentar, após o atingimento da maturidade sexual, uma melhoria da qualidade espermática [10,11].

\section{CONCLUSÃO}

Concluiu-se que machos suínos jovens com maiores taxas de crescimento corporal do nascimento aos $100 \mathrm{~kg}$ apresentam, na fase pós-púbere, performance sexual inferior frente a uma fêmea em estro e produção espermática semelhante a de animais com GPD inferior, porém com um maior porcentual de alterações da morfologia espermática.

\section{REFERÊNCIAS}

1 Cameron R.D.A. 1985. Measurement of semen production rates of boars. Australian Veterinary Journal. 62: 301-304.

2 Einarsson S., Holtman M., Larsson K., Settergren I. \& Bane A. 1979. The effect of two different feed levels on the development of the reproductive organs in boars. Acta Veterinaria Scandinavica. 20:1-9.

3 Flor-Cruz S.V. \& Lawood K.R. 1978. A longitudinal study of pubertal development in boars. International Journal of Andrology.1:317-330.

4 Hemsworth P.H., Beilharz R.G. \& Galloway D.B. 1977. Influence of social conditions during rearing on the sexual behavior of the domesticus boar. Animal Production. 24:245-251.

5 Hemsworth P.H. \& Findlay J.K. 1978. The importance of physical contact with other pigs during rearing on the sexual behavior of the male domestic pig. Animal Production. 27: 201-207.

6 Nelssen J.L., Davis D.L., Craig J.V. \& Hines R.H. 1982. Reproductive development in young boars exposed to sexually mature, nonpregnant sows and gilts. Theriogenology. 17: 545-550.

7 SAS users Guide: Statistics. 1985. Version 5 Edition. North Caroline, SAS Institut Inc. 956p.

8 Scheid I. R. \& Afonso S. B. 2000. Treinamento e manejo de machos suínos jovens e adultos. In: Anais do $7^{\circ}$ Simpósio Internacional de Reprodução e Inseminação Artificial em Suínos (Foz do Iguaçu, Brasil). pp. 29-39.

9 Scheid I.R., Wentz I., Mariano M. S. \& Silveira P. R. S. 1987. Quadro espermático de suínos em diferentes frequiências de ejaculação. In: Anais do $7^{\circ}$ Congresso Brasileiro de Reprodução (Belo Horizonte, Brasil). pp.60-61.

10 Swierstra E.E. 1973. Influence of breed, age, and ejaculation frequency on boar semen composition. Canadian Journal of Animal Science. 53: 43-53.

11 Swierstra E.E. 1974. A comparison of regular ejaculation with sexual rest on semen characteristics and reproductive organ weights in young boars. Journal of Animal Science. 39: 575-581.

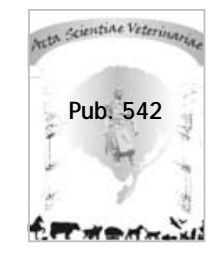

